\title{
LOCAL HOMEO- AND DIFFEOMORPHISMS: INVERTIBILITY AND CONVEX IMAGE
}

\author{
Gaetano Zampieri and Gianluca Gorni
}

\begin{abstract}
We prove a necessary and sufficient condition for a local homeomorphism defined on an open, connected subset of a Euclidean space to be globally one-to-one and, at the same time, for the image to be convex. Among the applications we give a practical sufficiency test for invertibility for twice differentiable local diffeomorphisms defined on a ball.
\end{abstract}

\section{INTRODUCTION}

A function between two topological spaces is said to be a local homeomorphism if it is continuous, open, and its restriction to a sufficiently small open neighbourhood of any point of its domain is one-to-one. The inverses of such restrictions will be called "local inverses" of the given function. For spaces with a differentiable structure, we speak of a local diffeomorphism if both the function and its local inverses are continuously differentiable. For reasonably well-behaved spaces, the local inverse theorem states that a continuously differentiable function is a local diffeomorphism if and only if the differential mapping is invertible at every point.

As is well known from the complex exponential, a local diffeomorphism needs by no means to be globally one-to-one. Characterisations of the class of the local homeomorphisms that are invertible in the large, or at least of large subclasses, have been given by many authors in different settings. Perhaps the neatest result along this line of research is that a necessary and sufficient condition for a local homeomorphism from a Banach space into another to be one-to-one and onto is that it be a proper map (that is, the inverse image of any compact set is compact), or, even less, a closed map (Hadamard [7], Levy [8], Caccioppoli [3], Banach and Mazur [1], Browder [2]). A few more recent papers and books on the subject are listed in the References, with no claim

Received 10th June, 1993.

Zampieri was supported during this research project by the Ministero della Università e della Ricerca Scientifica e Tecnologica (MURST). Gorni did most of his part in this work while visiting the Department of Mathematics of Chūō University, Tōkyō, supported by the MURST and by the "European Communities Scientific Training Programme in Japan" and is greatly indebted to Prof. Hitoshi Ishii and to all of Chūō University for their hospitality. The authors thank Prof. P.J. Rabier for some stimulating comments.

Copyright Clearance Centre, Inc. Serial-fee code: 0004-9729/94 \$A2.00+0.00. 
to completeness. The authors themselves were drawn to this area through their earlier interest in a problem in global asymptotic stability for ordinary differential equations in two dimensions, known as the Jacobian conjecture, which can be reformulated as a global invertibility problem for a local diffeomorphism (see [16]). For results and references on this conjecture and on a related one, bearing the same name, in algebraic geometry, see Meisters and Olech [9].

In the present work we add structure to the problem by concentrating on what happens if we ask a local homeomorphism or diffeomorphism defined on an open subset of a finite dimensional Euclidean space to be at once invertible and with convex image. Our basic, abstract result is Theorem 1.1 below. In the statement, for a function to be "locally convex" simply means that its restriction to a small enough convex neighbourhood of any point of the domain is a convex function.

THEOREM 1.1. Let $\Omega$ be a nonempty, open and connected subset of $\mathbb{R}^{n}$ and $f: \Omega \rightarrow \mathbb{R}^{n}$ be a local homeomorphism. Then the following two conditions are equivalent:

(a) $f$ is one-to-one and $f(\Omega)$ is convex;

(b) there exists a function $k: \Omega \rightarrow \mathbb{R}$ which is proper and bounded from below and whose composition with any local inverse of $f$ is a locally convex function.

The function $k$ will be hereby called an "auxiliary function". We shall see that, whenever the theorem applies, $k$ is necessarily continuous and that the function $f$ maps every set of the form $\{x \in \Omega: k(x)<c\}, c \in \mathbb{R}$, onto a convex set.

A first simple consequence of Theorem 1.1 follows by taking $f$ to be the identity mapping on $\Omega$ : a nonempty, open, connected subset of $\mathbb{R}^{n}$ is convex if and only if it is the domain of a proper, bounded from below and locally convex real function. It is easy to see that without properness the result does not hold.

If both $f$ and $k$ are smooth, the local convexity part of (b) translates into a pointwise condition involving the first- and second-order derivatives of $f$ and $k$ :

Proposition 1.2. Let $\Omega$ be a nonempty, open subset of $\mathbb{R}^{n}, f: \Omega \rightarrow \mathbb{R}^{n}$ be a $C^{2}$ local diffeomorphism and $k: \Omega \rightarrow \mathbb{R}$ be a $C^{2}$ function. Then the composition of $k$ with any local inverse of $f$ is locally convex if and only if for all $x \in \Omega$ the following quadratic form $Q_{x}$ is positive semidefinite:

$$
Q_{x}: \mathbb{R}^{n} \rightarrow \mathbb{R}, \quad Q_{x}(v):=k^{\prime \prime}(x)[v, v]-k^{\prime}(x) f^{\prime}(x)^{-1} f^{\prime \prime}(x)[v, v] .
$$

In the last formula the derivatives are to be viewed as multilinear mappings. In canonical co-ordinates of $\mathbb{R}^{n}$ and with the indexing and summation conventions of tensor calculus, the $i j$ entry $\left(Q_{x}\right)_{i j}$ of the $n \times n$ matrix representing the quadratic 
form $Q_{x}$ is

$$
\left(Q_{x}\right)_{i j}=\frac{\partial^{2} k}{\partial x^{i} \partial x^{j}}(x)-\frac{\partial k}{\partial x^{l}}(x)\left(f^{\prime}(x)^{-1}\right)_{m}^{l} \frac{\partial^{2} f^{m}}{\partial x^{i} \partial x^{j}}(x)
$$

The existence of a smooth $\left(C^{2}\right)$ auxiliary function for a given smooth local diffeomorphism is then equivalent to the system (1.2) of linear second-order partial differential equations in the unknowns $k$ and $\left(Q_{x}\right)_{i j}$ with the added conditions that the matrix $Q_{x}$ be symmetric and positive semidefinite all over $\Omega$ and the scalar function $k$ be bounded from below and proper. As a PDE problem it seems rather formidable, except in the one-dimensional case, where we shall show (Remark 5.3) that, given a $C^{2} f$ with nonzero first derivative on an interval, we can find an explicit solution of the (this time ODE) problem and even get $Q_{x}$ strictly positive. Of course, injectivity and convexity of the image are no issue in one dimension.

A more modest but feasible way of using Theorem 1.1 and Proposition 1.2 is to start from a given function $k$ and to look for practical conditions on a local diffeomorphism $f$ that ensure that $k$, or some variant of $k$, is an auxiliary function for $f$. The remaining results of this paper are all obtained by considering local diffeomorphisms defined on the open ball $B_{r_{0}}\left(x_{0}\right):=\left\{x \in \mathbb{R}^{n}:\left|x-x_{0}\right|<r_{0}\right\}$, and choosing $x \mapsto\left|x-x_{0}\right|^{2}$ and $x \mapsto 1 /\left(r_{0}^{2}-\left|x-x_{0}\right|^{2}\right)$ as $k$.

Propostition 1.3. Let $f: B_{r_{0}}\left(x_{0}\right) \rightarrow \mathbb{R}^{n}$ be a local $C^{2}$ diffeomorphism. Then the following two conditions are equivalent:

1. $f$ is one-to-one, the image $f\left(B_{r_{0}}\left(x_{0}\right)\right)$ is a convex set, and the function $y \mapsto\left|f^{-1}(y)-x_{0}\right|^{2}$ is convex on it;

2. the symmetric matrix

$$
I-\left(x-x_{0}\right) \cdot f^{\prime}(x)^{-1} f^{\prime \prime}(x)
$$

is positive semidefinite for all $x \in B_{r_{0}}\left(x_{0}\right)$.

Condition 2 is ensured in particular when the matrix is merely nonsingular everywhere on the ball.

In formula (1.3), as opposed to (1.1), we refer to the canonical co-ordinates and scalar product of $\mathbb{R}^{n} ; I-\left(x-x_{0}\right) \cdot f^{\prime}(x)^{-1} f^{\prime \prime}(x)$ is the $n \times n$ symmetric matrix associated with the quadratic form $v \mapsto|v|^{2}-\left(x-x_{0}\right) \cdot f^{\prime}(x)^{-1} f^{\prime \prime}(x)[v, v]$, whose $i j$ entry is

$$
\delta_{i j}-\left(x-x_{0}\right)^{p} \delta_{p l}\left(f^{\prime}(x)^{-1}\right)_{m}^{l} \frac{\partial^{2} f^{m}}{\partial x^{i} \partial x^{j}}(x)
$$

where $\delta$ is the Kronecker symbol. 
If convexity of the square norm of the inverse is of no interest in a given application, Proposition 1.3 can still be seen as a sufficient test of invertibility of a smooth mapping, based on the nonvanishing of two scalar functions: the determinant of the Jacobian matrix $f^{\prime}(x)$ and, if this is nonzero, determinant of the matrix $I-\left(x-x_{0}\right) \cdot f^{\prime}(x)^{-1} f^{\prime \prime}(x)$; the mapping $f$ will be one-to-one on the largest ball centred in $x_{0}$ where the two determinants are nonzero. It may be worthwhile to investigate whether an efficient numerical scheme can be developed from this idea.

A chapter in the theory of univalent holomorphic functions of one complex variable (see, for example, Duren [4]) is devoted to the holomorphic diffeomorphisms between an open disk of $\mathbb{C}$ and a convex set (in that context such transformations are called "convex functions"). With the usual identification of $\mathbb{C}$ with $\mathbb{R}^{2}$ we can translate our Proposition 1.3 into the following (apparently new) result in the theory of complex convex functions:

PROPOSITION 1.4. Let $f$ be a holomorphic function with everywhere nonvanishing first derivative defined on the disk $D_{r}=\{z \in \mathbb{C}:|z|<r\}, r>0$. Then the following two conditions are equivalent:

1. $f$ is one-to-one on $D_{r}$, the image $f\left(D_{r}\right)$ is a convex subset of $\mathbb{C}$ and the real function $w \mapsto\left|f^{-1}(w)\right|^{2}$ is convex on it;

2. the inequality $\left|f^{\prime}(z)\right| \geqslant\left|z f^{\prime \prime}(z)\right|$ holds for all $z \in D_{r}$.

This result isolates a subclass of the functions described by Theorem 2.11 of [4], which states that, with the same preamble as above, the next two conditions are equivalent:

$1^{\prime}$. $f$ is one-to-one on $D_{r}$ and the image $f\left(D_{r}\right)$ is a convex subset of $\mathbb{C}$;

$2^{\prime}$. the inequality $\Re\left(1+z f^{\prime \prime}(z) / f^{\prime}(z)\right)>0$ holds for all $z \in D_{r}$.

(The operator $\Re$ is the real part.) The maximal radius for condition 2 is obviously not larger than the maximal radius for condition $2^{\prime}$, and both are clearly positive. They coincide in the case of the complex exponential $f(z):=e^{z}$ and are distinct for $f(z):=1 /(1+z)$ (see Remark 5.5 for details).

Turning our attention back to Proposition 1.3, notice that its condition 2 is surely satisfied for a given $C^{2}$ local diffeomorphism $f$ whenever the radius $r$ is small enough, because the matrix $I-\left(x-x_{0}\right) \cdot f^{\prime}(x)^{-1} f^{\prime \prime}(x)$ reduces to $I$ when $x \rightarrow x_{0}$. All $C^{2}$ local diffeomorphisms then enjoy the property that for each $x_{0}$ there exists a convex neighbourhood of $f\left(x_{0}\right)$ where $f^{-1}$ is defined and on which the scalar function $y \mapsto$ $\left|f^{-1}(y)-x_{0}\right|^{2}$ is convex. Actually, without appealing to Proposition 1.3, this fact comes directly from the local inverse function theorem and the observation that the Hessian matrix of $y \mapsto\left|f^{-1}(y)-x_{0}\right|^{2}$ at $f\left(x_{0}\right)$ is $2\left(f^{\prime}\left(x_{0}\right)^{-1}\right)^{T} f^{\prime}\left(x_{0}\right)^{-1}$, which is positive definite. Less easy is to prove that the property holds with only $C^{1,1}$ regularity. 
This can be drawn from the more precise Proposition 1.5 below.

Proposition 1.5. Let $x_{0} \in \mathbb{R}^{n}, r>0$, and $f: B_{r}\left(x_{0}\right) \rightarrow \mathbb{R}^{n}$ be a local $C^{1}$ diffeomorphism. Suppose that there exist constants $0<M_{1} \leqslant M_{2}, L \geqslant 0$ such that

$$
\begin{gathered}
M_{1}|u| \leqslant\left|f^{\prime}(x)^{-1} u\right| \leqslant M_{2}|u| \\
\left|f^{\prime}\left(x_{1}\right)^{-1} u-f^{\prime}\left(x_{2}\right)^{-1} u\right| \leqslant L\left|x_{1}-x_{2}\right||u|
\end{gathered}
$$

for all $x, x_{1}, x_{2} \in B_{r}\left(x_{0}\right)$ and $u \in \mathbb{R}^{n}$. Suppose finally that $r \leqslant M_{1}^{2} / L M_{2}$. Then the mapping $f$ is one-to-one, the image $f\left(B_{r}\left(x_{0}\right)\right)$ is a convex set, and the function $y \leftrightarrow\left|f^{-1}(y)-x_{0}\right|^{2}$ is convex.

The one-variable $C^{1}$ diffeomorphism $f(x):=x+x|x|^{1 / 2}$ is not covered by the assumptions of Proposition 1.5, because it has infinite second derivative at $x=0$. Still, the function $y \mapsto\left|f^{-1}(y)\right|^{2}$ is convex in a neighbourhood of $y=0$. We could account for this by admitting the mapping $x \mapsto f^{\prime}(x)^{-1}$ in the proposition to be just locally Lipschitz continuous with constant $L(x)$ on the punctured ball $B_{r}\left(x_{0}\right) \backslash\left\{x_{0}\right\}$, provided that the product $\left|x-x_{0}\right| L(x)$ is small when $x \rightarrow x_{0}$, as it is the case in the example. Any attempt to further weaken the regularity cannot anyway lead to more than marginal improvements. The property breaks down for local diffeomorphisms that are merely $C^{1}$, as Remark 5.6 will indicate. This is not surprising, because a $C^{1}$ transformation cannot be hoped to preserve curvature.

The remainder of the paper is laid out as follows. In Section 2 we shall provide some preliminary facts on line-lifting as needed in our particular framework. For a much more general and abstract formulation see Rheinboldt [11]. In Section 3 we prove the part ' $(b) \Rightarrow(a)$ ' of Theorem 1.1. The main point will be to show that given $x_{0}, x_{1} \in \Omega$ there exists a continuous path $\gamma:[0,1] \rightarrow \Omega$ such that $\gamma(0)=x_{0}, \gamma(1)=x_{1}$ and that $f(\gamma(t))=t f\left(x_{1}\right)+(1-t) f\left(x_{0}\right)$ for all $t \in[0,1]$. In Section 4 the reverse implication '(a) $\Rightarrow(\mathrm{b})$ ' will be obtained by constructing a function $K: f(\Omega) \rightarrow \mathbb{R}$ which is convex, proper and bounded from below and by defining the auxiliary function as $k:=K \circ f$.

In Section 5 we shall collect miscellaneous remarks and the proofs of the propositions that we have listed above. Finally, Section 6 is devoted to the ideas from differential geometry that originally inspired this work: a theorem of Gordon's on geodesic connectivity, as applied to a suitable Riemannian metric on $\Omega$ that can be associated with a given local diffeomorphism. In that setting the otherwise obscure quadratic form $Q_{x}$ of Proposition 1.2 will be given a simple differential-geometric interpretation.

\section{LINE-LIFTING}

Line-lifting is the implicit function problem of finding continuous paths $\gamma$ in $\Omega$ that emanate from a given point $x_{0} \in \Omega$ and are mapped into straight lines by the 
function $f: \Omega \rightarrow \mathbb{R}^{n}:$

$$
\gamma(0)=x_{0}, \quad f(\gamma(t))=f(x)+t v
$$

for a given $v \in \mathbb{R}^{n}$.

A first reading of this section may be expedited by assuming $f$ to be a local diffeomorphism, so that $\gamma$ is differentiable and we can take the derivative of equation (2.1) with respect to $t$ and look for $\gamma$ as a solution of a Cauchy problem for a differential equation

$$
\gamma^{\prime}(t)=f^{\prime}(\gamma(t))^{-1} v, \quad \gamma(0)=\dot{x_{0}}
$$

(Ważewski's equation; see [13]) instead of a solution to an implicit function problem. It will be easily seen that, with $C^{2}$ regularity on $f$, Propositions 2.2 and 2.3 become very standard results in the theory of ordinary differential equations. The differential equation point of view results in a loss of regularity, however, because if $f$ is a $C^{m}$ local diffeomorphism, $m \geqslant 1, \gamma(t)$ actually depends in a $C^{m}$ way on the triple $(x, v, t)$, and not just $C^{m-1}$, as one would be led to believe by the general theory of ODE applied to $(2.2)$.

In Section 6 we shall see that, with suitable regularity of $f$, the paths $\gamma$ can also be seen as geodesic curves for a Riemannian structure on $\Omega$ induced by $f$.

In the rest of this section, $\Omega$ will be a nonempty, open and connected subset of $\mathbb{R}^{n}$ and $f: \Omega \rightarrow \mathbb{R}^{n}$ a local homeomorphism.

Definition 2.1: Given $x \in \Omega, v \in \mathbb{R}^{n}$, we shall call a "line-lifting path" a continuous function $\gamma: I \rightarrow \Omega$, defined on an interval $I \subset \mathbb{R}$ containing 0 , such that

$$
f(\gamma(t))=f(x)+t v \quad \text { for all } t \in I .
$$

Even without using the continuity of $f$, we can say that the set of line-lifted paths is nonempty, because it contains the trivial $\gamma(t) \equiv x$ defined on the degenerate interval $I=\{0\}$. The set is also naturally endowed with the partial ordering defined by the restriction of functions. Zorn's lemma allows us to say that every line-lifting path is then the restriction of a maximal path, that is, of a path that is not itself the restriction of a line-lifting path defined on a strictly larger interval. Of course, in our setting we can say much more. We omit the proofs of the next two propositions, which only involve basic topology. The first one concentrates on the dependence of $\gamma$ from $t$, while the second one is about the joint dependence on $(t, x, v)$.

Proposition 2.2. For any given $x \in \Omega, v \in \mathbb{R}^{n}$, there exists one and only one maximal line-lifting path $\gamma_{x, v}$ defined on the interval $I_{x, v}$. Moreover, $\gamma_{x, v}(t)$ only 
depends on the product $t v$ : if $t \in I_{x, v}, t^{\prime} \in \mathbb{R}, v^{\prime} \in \mathbb{R}^{n}$ and $t^{\prime} v^{\prime}=t v$ then $t^{\prime} \in I_{x, v^{\prime}}$ and $\gamma_{x, v^{\prime}}\left(t^{\prime}\right)=\gamma_{x, v}(t)$.

Proposition 2.3. The set $D:=\left\{(x, v, t): x \in \Omega, v \in \mathbb{R}^{n}, t \in I_{x, v}\right\}$ is open in $\Omega \times \mathbb{R}^{n} \times \mathbb{R}$, the function $(x, v, t) \mapsto \gamma_{x, v}(t)$ is continuous on $D$, and for any fixed $x \in \Omega, t \neq 0$, the mapping $v \mapsto \gamma_{x, v}(t)$ is an open mapping on $\left\{v \in \mathbb{R}^{n}:(x, v, t) \in D\right\}$.

When $f$ is a $C^{m}$ local diffeomorphism, $m \geqslant 1, \gamma_{x, v}(t)$ is also $C^{m}$ regular in the triple $(x, v, t)$, and the openness of the mapping $v \mapsto \gamma_{x, v}(t)$ for $t \neq 0$ can be easily obtained by differentiating formula (2.1) with respect to $v$ :

$$
f^{\prime}\left(\gamma_{x, v}(t)\right) D_{v} \gamma_{x, v}(t)=t I,
$$

(where $I$ is the identity mapping in $\mathbb{R}^{n}$ ) and noticing that $D_{v} \gamma_{x, v}(t)$ is invertible.

The following propositions uses the fact that we are lifting straight lines and not just continuous paths.

Proposition 2.4. Let $C$ be a compact subset of $\Omega, x \in \Omega$ and $v \in \mathbb{R}^{n}$, $v \neq 0$. Then there exists $\bar{t} \in I_{x, v}$ such that $\gamma_{x, v}(t) \in \Omega \backslash C$ for all $t \in I_{x, v}, t \geqslant \bar{t}$.

Proof: Suppose first that $\sup I_{x, v}=+\infty$. Since $f(C)$ is compact and $v \neq 0$, there exists $\bar{t} \geqslant 0$ such that

$$
f(x)+t v \in \mathbb{R}^{n} \backslash f(C) \quad \text { for all } t \geqslant \bar{t} .
$$

But $f(x)+t v=f\left(\gamma_{x, v}(t)\right)$ for all $t \geqslant 0$, whence the conclusion. Suppose instead that $\sup I_{x, v}<+\infty$ and that the property were not true. Then there would exist a sequence of times $t_{m}$ such that

$$
t_{m} \nearrow \sup I_{x, v}<+\infty, \quad \gamma_{x, v}\left(t_{m}\right) \in C .
$$

Of course we can suppose that the sequence $\gamma_{x, v}\left(t_{m}\right)$ converges to a point $y \in C$. Let $U_{y}^{\prime} \subset U_{y}$ be two neighbourhoods of $y$ in $\Omega$ where $f$ is one-to-one, with $U_{y}$ open and $U_{y}^{\prime}$ compact. We can safely assume that $\gamma_{x, v}\left(t_{m}\right) \in U_{y}^{\prime}$ for all $m \in \mathbb{N}$. In particular

$$
f\left(\gamma_{x, v}\left(t_{m}\right)\right)=f(x)+t_{m} v \in f\left(U_{y}^{\prime}\right) .
$$

Since the compact set $f\left(U_{y}^{\prime}\right)$ is disjoint from the set $\mathbb{R}^{n} \backslash f\left(U_{y}\right)$, which is closed in $\mathbb{R}^{n}$, their distance is positive:

$$
\delta:=\operatorname{dist}\left(f\left(U_{y}^{\prime}\right), \mathbb{R}^{n} \backslash f\left(U_{y}\right)\right)>0 .
$$

The point $f(x)+t v$ cannot move from $f\left(U_{y}^{\prime}\right)$ to $\mathbb{R}^{n} \backslash f\left(U_{y}\right)$ in less than $\delta /|v|$ units of time:

$$
\left|t-t_{m}\right|<\frac{\delta}{|v|} \Rightarrow f(x)+t v \in f\left(U_{y}\right)
$$


and, until it leaves $f\left(U_{y}\right)$, the line-lifting is defined as

$$
\gamma_{x, v}(t):=\left.f\right|_{U_{y}} ^{-1}(f(x)+t v) \in U_{y} .
$$

Hence for all $m \in \mathbb{N}$ the whole interval $\left[t_{m}, t_{m}+\delta|v|^{-1}\right)$ is contained in $I_{x, v}$, which contradicts our assumption that $t_{m} \nearrow \sup I_{x, v}<+\infty$.

\section{Proof of Theorem 1.1, Part I}

Let $\Omega$ be a nonempty, open and connected subset of $\mathbb{R}^{n}, f: \Omega \rightarrow \mathbb{R}^{n}$ be a local homeomorphism and $k: \Omega \rightarrow \mathbb{R}$ be a function. It is easy to see how the local convexity mentioned in condition (b) of Theorem 1.1 relates to the line-lifting paths $\gamma_{x, v}(t)$ introduced in Section 2. Namely, the following two conditions are equivalent:

(i) the composition of $k$ with any local inverse of $f$ is a locally convex function;

(ii) for any $x \in \Omega, v \in \mathbb{R}^{n}$ the function $t \mapsto k\left(\gamma_{x, v}(t)\right)$ is convex on $I_{x, v}$.

We are now ready to tackle the implication ' $(b) \Rightarrow(a)$ ' of Theorem 1.1.

Proof of Theorem 1.1, (b) $\Rightarrow$ (a): The problem can be reduced to proving that, for any pair of points $x, y \in \Omega$, the straight line segment joining $f(x)$ with $f(y)$ is the image through $f$ of a continuous path in $\Omega$ joining $x$ with $y$. It will then be obvious that $f(\Omega)$ is convex. As for the invertibility of $f$, if $f(x)=f(y)$ then $x$ and $y$ will be connected in $\Omega$ by a continuous path along which $f$ is constant. This is only possible if $x=y$ because $f$ is a local homeomorphism.

Within the framework introduced in Section 2, we must show that for any $x, y \in \Omega$ there exist $v \in \mathbb{R}^{n}, t \in I_{x, v}$ such that $\gamma_{x, v}(t)=y$. Let $x$ be fixed and consider the set

$$
R:=\left\{\gamma_{x, v}(t): v \in \mathbb{R}^{n}, t \in I_{x, v}\right\} \subset \Omega
$$

We shall conclude that $R=\Omega$ if we are able to prove that $R$ is nonempty, open and closed in the connected set $\Omega$. Obviously $x \in R$ because $t \in I_{x, v}$ and $\gamma_{x, v}(t)=x$ whenever $t v=0$ (take for example $v=0$ and $t \in I_{x, 0}=\mathbb{R}$ arbitrary).

To see that $R$ is open, let us note that we can restrict $t$ to be nonzero in the definition of $R$, because $x$ is recovered anyway, and then $R$ can be written as the union of a family of sets

$$
R=\bigcup_{t \neq 0}\left\{\gamma_{x, v}(t): v \in \mathbb{R}^{n}, t \in I_{z, v}\right\}
$$

each of which is open because the mapping $v \mapsto \gamma_{z, v}(t)$ is open on the open set $\{v \in$ $\left.\mathbb{R}^{n}: t \in I_{x, v}\right\}$ if $t \neq 0$ (Proposition 2.3). 
We are left to verify whether $R$ is closed in $\Omega$. Let $y_{m}=\gamma_{x, v_{m}}\left(t_{m}\right)$ be a sequence of points in $R$ converging to $y \in \Omega$. The question is whether the respective paths converge, too. Cannot they conceivably drift to the boundary of $\Omega$ or to infinity, instead, as $m \rightarrow+\infty$ ? The auxiliary function $k$ is what will rule out this possibility. But first some normalisation of parameters. Since

$$
f\left(\gamma_{x, v_{m}}\left(t_{m}\right)\right)=f(x)+t_{m} v_{m} \rightarrow f(y)
$$

the sequence $t_{m} v_{m}$ converges to $f(y)-f(x)$. Upon a linear reparameterisation of the paths, we can safely assume that $t_{m} \equiv 1$ and $v_{m} \rightarrow \bar{v}:=f(y)-f(x)$ (by Proposition 2.2 it suffices to take $v_{m}^{\prime}=t_{m} v_{m}$ instead of $v_{m}$, because $\gamma_{x, v_{m}^{\prime}}(1)=y_{m}$ too).

The problem further reduces to proving that $1 \in I_{x, \bar{v}}$, because if this is the case then Proposition 2.3 will give that

$$
y=\lim _{m \rightarrow+\infty} \gamma_{x, v_{m}}(1)=\gamma_{x, \bar{v}}(1) \in R
$$

At last we come to exploit the auxiliary function $k$. From the local convexity property it follows that the set of the times $t$ for which a generic line-lifting path $\gamma_{x, v}(t)$ belongs to a set of the form $L_{c}:=\left\{z \in \mathbb{R}^{n}: k(z) \leqslant c\right\}$, for $c \in \mathbb{R}$, is an interval. In fact, since the function $h(t):=k\left(\gamma_{x, v}(t)\right)$ is locally convex on $I_{x, v}$, it is globally convex, so that from $h\left(t^{\prime}\right) \leqslant c, h\left(t^{\prime \prime}\right) \leqslant c$ we deduce that $h(t) \leqslant c$ for all the $t$ in between $t^{\prime}$ and $t^{\prime \prime}$.

Let us specialise $c$ to be the (finite) supremum of the continuous function $k$ over the compact set $\left\{y_{m}: m \in \mathbb{N}\right\} \cup\{x, y\} \subset \Omega$. We can say that the relation $\gamma_{x, v_{m}}(t) \in L_{c}$ holds for all $t \in[0,1]$, because it does for both $t=0$ and $t=1$.

From the hypothesis (b) we have that $L_{c}$ is compact. From Proposition 2.4 we are assured that the path $\gamma_{x, \bar{v}}$ will eventually escape from $L_{c}$ : there exists $\bar{t} \in I_{x, \bar{v}}, \bar{t} \geqslant 0$ such that $\gamma_{x, \bar{v}}(\bar{t}) \in \Omega \backslash L_{c}$. But then $\bar{t} \in I_{x, v_{m}}$ and $\gamma_{x, v_{m}}(\bar{t})$ is in the open set $\Omega \backslash L_{c}$ for all large $m$ (Proposition 2.3). Thus $\bar{t}>1$ and we are done.

\section{Proof of TheOrem 1.1 , Part II}

Given two nonempty open sets $\Omega, C$ in $\mathbb{R}^{n}$, of which $C$ is convex, and a homeomorphism $f: \Omega \rightarrow C$, we wish to prove that there exists an auxiliary function for $f$, that is to say, a proper, bounded from below function $k: \Omega \rightarrow \mathbb{R}$ for which the composition $k \circ f^{-1}$ is a convex function. Notice that the function $K:=k \circ f^{-1}$ will itself be proper, bounded from below, beside being convex: it will be an auxiliary function associated with the identity mapping $C \rightarrow C$. The other way round, if we come up with a proper, bounded from below and convex $K: C \rightarrow \mathbb{R}$, then an auxiliary function 
for a general $f: \Omega \rightarrow C$ will simply be $k \equiv K \circ f$ :

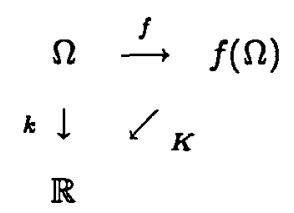

We can then forget about all the data of the problem except for the set $C$. The purpose will be to define a convex function $K: C \rightarrow \mathbb{R}$ which is proper and bounded from below.

The special case when $C=\mathbb{R}^{n}$ is very easy: we can simply define $K(y):=|y|$. This $K$ is obviously convex, bounded from below, and the inverse image of a compact subset of $\mathbb{R}$ is itself compact because it is bounded and closed in $\mathbb{R}^{n}$.

To tackle the general case, we start with recalling some well-known properties of the gauge function $j_{C}: \mathbb{R}^{n} \rightarrow \mathbb{R}$ associated to an open, convex subset $C$ of $\mathbb{R}^{n}$ containing 0 :

$$
j_{C}(x):=\inf \{\lambda>0: x / \lambda \in C\} \quad \text { for } x \in \mathbb{R}^{n} .
$$

Since 0 is in the interior of $C$, there exists $\varepsilon>0$ such that $\varepsilon x /|x| \in C$ for any $x \neq 0$. It follows readily that $0 \leqslant j_{C}(x) \leqslant|x| / \varepsilon$ for all $x \in \mathbb{R}^{n}$. It is also clear that $j_{C}$ is positively homogeneous of degree 1 . Next, if $x, y \in \mathbb{R}^{n}$ and $\lambda, \mu>0$, we can write

$$
\left(\frac{x}{\lambda} \in C, \frac{y}{\mu} \in C\right) \Rightarrow \frac{x+y}{\lambda+\mu}=\frac{\lambda}{\lambda+\mu}\left(\frac{x}{\lambda}\right)+\frac{\mu}{\lambda+\mu}\left(\frac{y}{\mu}\right) \in C,
$$

so that $j_{C}$ is subadditive: $j_{C}(x+y) \leqslant j_{C}(x)+j_{C}(y)$ for any $x, y \in \mathbb{R}^{n}$. Since it is homogeneous and subadditive, it must be convex too. Lipschitz continuity also follows easily from the relation

$$
\begin{aligned}
j_{C}(y)-j_{C}(x)=j_{C}(y-x+x)- & j_{C}(x) \leqslant j_{C}(y-x)+j_{C}(x)-j_{C}(x) \\
& =j_{C}(y-x) \leqslant \frac{|y-x|}{\varepsilon} \quad \text { for any } x, y \in \mathbb{R}^{n}
\end{aligned}
$$

and the one obtained by exchanging $x$ and $y$.

The gauge function $j_{C}$ completely describes the set $C$ :

$$
C=\left\{x \in \mathbb{R}^{n}: j_{C}(x)<1\right\}, \quad \partial C=\left\{x \in \mathbb{R}^{n}: j_{C}(x)=1\right\} .
$$

In fact, if $x \in C$ then $(1+\delta) x \in C$ for $\delta>0$ small enough, so that $j_{C}(x) \leqslant(1+\delta)^{-1}<$ 1. Conversely, if $j_{C}(x)<1$ then $x / \lambda \in C$ for some some $\lambda<1$ and $x$ also belongs to $C$ because it lies on the segment joining $0 \in C$ with $x / \lambda$. To prove the " $C$ " inclusion in the formula for $\partial C$ it is now enough to notice that $j_{C}(x) \leqslant 1$ for all $x$ in the closure 
of $C$ because $j_{C}$ is continuous and that $j_{C}(x)<1$ only for the $x$ in $C$, which does not intersect $\partial C$. Conversely, if $j_{C}(x)=1$ then $\mu x \in C$ for all $\mu \in[0,1)$ because $j_{C}(\mu x)=\mu j_{C}(x)<1$, and $x=\lim _{\mu / 1} \mu x$ must be in the closure of $C$, but not in $C$ itself.

The following lemma on convex functions is straightforward.

LEMma 4.1. Let $D \subset \mathbb{R}^{n}$ be a nonempty, convex set, $h: D \rightarrow \mathbb{R}$ be a convex function and $\varphi: \mathbb{R} \rightarrow \mathbb{R} \cup\{+\infty\}$ be a weakly increasing, convex function. Then the composition $\varphi \circ h: D \rightarrow \mathbb{R} \cup\{+\infty\}$ is a convex function.

We are now ready to complete the proof of Theorem 1.1.

Proof of Theorem 1.1, (a) $\Rightarrow(\mathrm{b})$ : As we have remarked, we only need prove that for any open, convex set $C$ in $\mathbb{R}^{n}$ there is a convex function $K: C \rightarrow \mathbb{R}$ which is proper and bounded from below, two conditions that together mean, roughly speaking, that $K$ must diverge to $+\infty$ on the boundary of $C$ (if $C \neq \mathbb{R}^{n}$ ) and as $|y| \rightarrow+\infty$ (if $C$ is unbounded). Since $C$ is open in $\mathbb{R}^{n}$ and it is not restrictive to assume that $0 \in C$, we can consider the gauge function $j_{C}: \mathbb{R}^{n} \rightarrow \mathbb{R}$ associated with $C$. We know that $j_{C}$ is convex, continuous and $0 \leqslant j_{C}(y)<1 \Longleftrightarrow y \in C, j_{C}(y)=1 \Longleftrightarrow y \in \partial C$. It is not difficult to coerce $j_{C}$ to diverge on the boundary of $C:$ it is enough to compose it with a function of one variable which is finite on $[0,1)$ and that diverges at 1 . Of course, we must also make sure not to lose convexity and continuity. The function $t \mapsto 1 /(1-t)$ works out, because it is continuous, increasing and convex on the interval $[0,1)$ and it diverges to $+\infty$ as $t / 1$. Hence the function $\widetilde{K}$ defined as

$$
\tilde{K}(y):=\frac{1}{1-j_{C}(y)} \quad \text { for } y \in C
$$

is continuous and convex on $C$ from Lemma 4.1. It is also $\geqslant 1$.

To ensure that $K$ diverges also as $|y| \rightarrow+\infty$, we define it as:

$$
K(y):=|y|+\widetilde{K}(y) \quad \text { for } y \in C .
$$

This $K$ is convex and continuous on $C$ because it is the sum of two functions with those same properties. It is also $\geqslant 1$. For $c>0$ the set $\{y \in C: K(y) \leqslant c\}=K^{-1}((-\infty, c])$ is closed in $C$ and contained in a closed subset of $\mathbb{R}^{n}$ :

$$
\begin{aligned}
\{y \in C: K(y) \leqslant c\} & \subset\{y \in C: \tilde{K}(y) \leqslant c\} \\
& =\left\{y \in \mathbb{R}^{n}: j_{C}(y) \leqslant 1-\frac{1}{c}\right\}=j_{C}^{-1}\left(\left(-\infty, 1-\frac{1}{c}\right]\right) .
\end{aligned}
$$

Then it is itself closed in $\mathbb{R}^{n}$. But it is also bounded because

$$
\{y \in C: K(y) \leqslant c\} \subset\left\{y \in \mathbb{R}^{n}:|y| \leqslant c\right\} .
$$


Hence $\{y \in C: K(y) \leqslant c\}$ is compact for $c>0$. This property readily takes care of both properness and boundedness from below.

Observe that if $C$ is bounded, that is, there exists $M>0$ such that $|y| \leqslant M$ for all $y \in C$, then $j_{C}(y) \geqslant|y| / M$ for all $y \in \mathbb{R}^{n}$ and the set $\{y \in C: \widetilde{K}(y) \leqslant c\}$ is bounded. In this case we could more simply define $K \equiv \widetilde{K}$.

\section{OTHER PROOFS AND REMARKS}

REMARK 1. The auxiliary function $k$ in condition (b) of Theorem 1 is necessarily continuous. Suppose in fact that $k: \Omega \rightarrow \mathbb{R}$ is a function whose composition with any local inverse of $f$ is a locally convex function. We want to show that $k$ is continnous. Let then $y=f(x) \in f(\Omega), U$ be an open neighbourhood of $x$ in $\Omega$ where $f$ is invertible and such that $V:=f(U)$ is convex and $K:=\left.k \circ f\right|_{U} ^{-1}$ is a convex function. We shall be done if $K$ turns out to be continuous, because then $\left.k\right|_{U}=\left.K \circ f\right|_{U}$ will be continuous. But $K$ is convex and finite on the open, convex set $V$, and the continuity follows from a well-known property of convex functions. Actually, $k$ must be locally Lipschitz whenever $f$ is.

REMARK 5.2. Suppose that Theorem 1 holds for a function $f: \Omega \rightarrow \mathbb{R}^{n}$ and an auxiliary function $k: \Omega \rightarrow \mathbb{R}$. Then the set $f(\{x \in \Omega: k(x)<c\})$ is convex for any $c \in \mathbb{R}$. Suppose in fact $x_{1}, x_{2} \in \Omega, k\left(x_{1}\right)<c, k\left(x_{2}\right)<c, \theta \in[0,1]$. Define $x(\theta):=f^{-1}\left(\theta f\left(x_{1}\right)+(1-\theta) f\left(x_{2}\right)\right)$. We must prove that $k(x(\theta))<c$. But this is easy because $k \circ f^{-1}: f(\Omega) \rightarrow \mathbb{R}$ is a convex function (being locally convex on a convex set), so that we can write

$$
\begin{aligned}
k(x(\theta)) & =k \circ f^{-1}\left(\theta f\left(x_{1}\right)+(1-\theta) f\left(x_{2}\right)\right) \\
& \leqslant \theta\left(k \circ f^{-1}\right)\left(f\left(x_{1}\right)\right)+(1-\theta)\left(k \circ f^{-1}\right)\left(f\left(x_{2}\right)\right) \\
& =\theta k\left(x_{1}\right)+(1-\theta) k\left(x_{2}\right)<c .
\end{aligned}
$$

The set $\{x \in \Omega: k(x)<c\}$ is connected, in particular. Theorem 1 applies on it with $x \mapsto 1 /(c-k(x))$ as auxiliary function.

Proof of Proposition 1.2: We are only dealing with local properties, and we can simplify the notation by assuming $f$ to be invertible on $\Omega$. Since $k \circ f^{-1}$ is a $C^{2}$ function, saying that it is locally convex is the same as saying that for any $x \in \Omega$ and $u \in \mathbb{R}^{n}$ the second derivative at $t=0$ of the real function $t \mapsto\left(k \circ f^{-1}\right)(f(x)+t u)$ is $\geqslant$ 0 . Define $\gamma(t):=f^{-1}(f(x)+t u)$. Let us compute the first derivative for arbitrary $t$ :

$$
\frac{d}{d t}\left(k \circ f^{-1}\right)(f(x)+t u)=\frac{d}{d t} k(\gamma(t))=k^{\prime}(\gamma(t)) \gamma^{\prime}(t)=k^{\prime}(\gamma(t)) f^{\prime}(\gamma(t))^{-1} u
$$


and the second derivative, using the formula for the derivative of the inverse matrix:

$$
\begin{aligned}
\frac{d^{2}}{d t^{2}}\left(k \circ f^{-1}\right)(f(x)+t u)= & k^{\prime \prime}(\gamma(t))\left[\gamma^{\prime}(t), \gamma^{\prime}(t)\right] \\
& -k^{\prime}(\gamma(t)) f^{\prime}(\gamma(t))^{-1}\left(\frac{d}{d t} f^{\prime}(\gamma(t))\right) f^{\prime}(\gamma(t))^{-1} v \\
= & k^{\prime \prime}(\gamma(t))\left[\gamma^{\prime}(t), \gamma^{\prime}(t)\right] \\
& -k^{\prime}(\gamma(t)) f^{\prime}(\gamma(t))^{-1} f^{\prime \prime}(\gamma(t))\left[\gamma^{\prime}(t), \gamma^{\prime}(t)\right]
\end{aligned}
$$

By setting $t=0$ and $v:=\gamma^{\prime}(t)=f^{\prime}(x)^{-1} u$ we get

$$
\left.\frac{d^{2}}{d t^{2}}\left(k \circ f^{-1}\right)(f(x)+t u)\right|_{t=0}=k^{\prime \prime}(x)[v, v]-k^{\prime}(x) f^{\prime}(x)^{-1} f^{\prime \prime}(x)[v, v] .
$$

The vector $v$ can be anywhere in $\mathbb{R}^{n}$ because $f^{\prime}(x)$ is invertible, and the conclusion follows. If desired, the right-hand side of formula (5.4) also gives the second differential $\left(k \circ f^{-1}\right)^{\prime \prime}(f(x))[u, u]$ of the composition $k \circ f^{-1}$, by substituting back $f^{\prime}(x)^{-1} u$ for $v$.

Proof of Proposition 1.3: Suppose that $f$ is one-to-one on $B_{r_{0}}\left(x_{0}\right)$ and that $y \mapsto\left|f^{-1}(y)-x_{0}\right|^{2}$ is locally convex. Then Proposition 1.3 can be applied to the scalar function $\widetilde{k}(x):=\left|x-x_{0}\right|^{2}$, yielding that

$$
\widetilde{Q}_{x}(v):=|v|^{2}-\left(x-x_{0}\right) \cdot f^{\prime}(x)^{-1} f^{\prime \prime}(x)[v, v] \geqslant 0
$$

for all $x \in B_{r_{0}}\left(x_{0}\right), v \in \mathbb{R}^{n}$, which is equivalent to positive semidefiniteness of the matrix (1.3). Conversely, suppose that inequality (5.5) holds. The composition of $\widetilde{k}$ with any local inverse of $f$ will then be locally convex, because of Proposition 1.3 again. Note that such composition takes values in the interval $\left[0, r_{0}^{2}\right)$, and that the function $g(\sigma):=1 /\left(r_{0}^{2}-\sigma\right)$ is convex and increasing on that interval. Define $k:=$ $g \circ \tilde{k}$. Then also the composition of $k$ with any local inverse of $f$ is locally convex, but this $k$ is also bounded from below and proper on $B_{r_{0}}\left(x_{0}\right)$. From Theorem 1.1 we deduce that $f$ is one-to-one and that $f\left(B_{r_{0}}\left(x_{0}\right)\right)$ is convex. The fact that $y \mapsto$ $\left|f^{-1}(y)-x_{0}\right|^{2}=\left(\tilde{k} \circ f^{-1}\right)(y)$ is convex follows from formula (5.5) and Proposition 1.3 once more. Finally, if the symmetric matrix $I-\left(x-x_{0}\right) \cdot f^{\prime}(x)^{-1} f^{\prime \prime}(x)$ associated with the quadratic form $\widetilde{Q}_{x}$ is everywhere nonsingular, it is positive semidefinite if and only if it is positive definite. The set of the $x \in B_{r_{0}}\left(x_{0}\right)$ for which $\widetilde{Q}_{x}$ is positive (semi-) definite is nonempty (because it surely contains $x_{0}$ ), closed and open and the proof is complete. 
REMARK 5.3. Let $I$ be a nonempty, open interval in $\mathbb{R}, f: I \rightarrow \mathbb{R}$ be a $C^{2}$ function with an everywhere positive first derivative. Then there exists a $C^{2}$ function $k: I \rightarrow \mathbb{R}$ and a continuous $Q: I \rightarrow \mathbb{R}$ such that

$$
\begin{aligned}
& k^{\prime \prime}-k^{\prime} \frac{f^{\prime \prime}}{f^{\prime}}=Q \text { in } I, \quad Q>0 \text { in } I, \\
& \lim _{x \backslash \text { inf } I} k(x)=\lim _{x / \sup I} k(x)=+\infty .
\end{aligned}
$$

In fact, the first relation can be regarded as a nonhomogeneous first-order linear ordinary differential equation in $k$, a particular solution of which is

$$
k(x)=\int_{x_{0}}^{x}\left(f^{\prime}(s) \int_{x_{0}}^{s} \frac{Q(t)}{f^{\prime}(t)} d t\right) d s
$$

where $x_{0} \in I$ is arbitrary. Let $\tilde{k}: I \rightarrow \mathbb{R}$ be a $C^{2}$ function such that

$$
\tilde{k}^{\prime \prime}>0 \quad \text { in } I, \quad \tilde{k}^{\prime}\left(x_{0}\right)=0, \quad \lim _{x \in \inf I} \tilde{k}(x)=\lim _{x / \text { sup } I} \tilde{k}(x)=+\infty,
$$

and $h: I \rightarrow \mathbb{R}$ be a $C^{1}$ function such that

$$
h(x) \geqslant \frac{1}{f^{\prime}(x)} \quad \text { for all } x \in I, \quad h^{\prime}(x) \begin{cases}>0 & \text { if } x>x_{0}, \\ <0 & \text { if } x<x_{0} .\end{cases}
$$

Define

$$
Q(x)=f^{\prime}(x) \frac{d}{d x}\left(h(x) \tilde{k}^{\prime}(x)\right)=f^{\prime}(x)\left(h^{\prime}(x) \widetilde{k}^{\prime}(x)+h(x) \widetilde{k}^{\prime \prime}(x)\right)
$$

This $Q$ is positive and continuous. The corresponding $k^{\prime}$ becomes

$$
k^{\prime}(x)=f^{\prime}(x) h(x) \tilde{k}^{\prime}(x) \begin{cases}\geqslant \tilde{k}^{\prime}(x) & \text { if } x \geqslant x_{0}, \\ \leqslant \tilde{k}^{\prime}(x) & \text { if } x \leqslant x_{0}\end{cases}
$$

whence

$$
k(x) \geqslant \tilde{k}(x)-\tilde{k}\left(x_{0}\right) \rightarrow+\infty \quad \text { both as } x \nearrow \sup I \text { and as } x \searrow \inf I .
$$

Proof of Proposition 1.4: With the usual identification of $z \in \mathbb{C}$ and $x \in \mathbb{R}^{2}$, the matrix $I-x \cdot \widetilde{f}(x)^{-1} \tilde{f}^{\prime \prime}(x)$ of Proposition 1.3 has $1 \pm|w|$, with $w=\bar{z} f^{\prime \prime}(z) / f^{\prime}(z)$, as eigenvalues because of the following Lemma 5.4. These two eigenvalues are nonnegative if and only if $|w| \leqslant 1$, which is equivalent to condition 2 . 
Lemma 5.4. Let $\Omega$ be an open set in $\mathbb{C}$ and $f: \Omega \rightarrow \mathbb{C}$ be a holomorphic function. Let $\tilde{f}$ be the real version of $f$. Then, for all $z=x_{1}+i x_{2} \in \Omega, x=$ $\left(x_{1}, x_{2}\right)^{T} \in \mathbb{R}^{2}$, if $f^{\prime}(z) \neq 0$ we have

$$
x \cdot \tilde{f}^{\prime}(x)^{-1} \tilde{f}^{\prime \prime}(x)=\left(\begin{array}{cc}
\Re w & -\Im w \\
-\Im w & -\Re w
\end{array}\right), \quad \text { with } w=\frac{\bar{z} f^{\prime \prime}(z)}{f^{\prime}(z)} .
$$

This matrix has $\pm|w|$ as eigenvalues.

Proof: We must of course use the symmetries that the holomorphy of $f$ generates in the first and second differentials of $\tilde{f}$. First, by comparing the second order terms $f^{\prime \prime}(z) w^{2} / 2$ and $\widetilde{f}^{\prime \prime}(x)[v, v] / 2$ in the Taylor expansions respectively of $f$ at $z$ and of $\widetilde{f}$ at $x$, having identified $w=v_{1}+i v_{2} \in \mathbb{C}, v=\left(v_{1}, v_{2}\right)^{T} \in \mathbb{R}^{2}$, we see that

$$
\tilde{f}^{\prime \prime}(x)[v, v]=\left(\begin{array}{cc}
\Re f^{\prime \prime}(z) & -\Im f^{\prime \prime}(z) \\
\Im f^{\prime \prime}(z) & \Re f^{\prime \prime}(z)
\end{array}\right)\left(\begin{array}{c}
v_{1}^{2}-v_{2}^{2} \\
2 v_{1} v_{2}
\end{array}\right) .
$$

Applying $\tilde{f}^{\prime}(x)^{-1}$ to the left will produce the real matrix $\left(\begin{array}{cc}a & -b \\ b & a\end{array}\right)$ associated with the complex product with $f^{\prime \prime}(z) / f^{\prime}(z)=a+i b, a, b \in \mathbb{R}$. We can write

$$
\begin{aligned}
x \cdot \tilde{f}^{\prime}(x)^{-1} f^{\prime \prime}(x)[v, v] & =\left(x_{1}, x_{2}\right)\left(\begin{array}{cc}
a & -b \\
b & a
\end{array}\right)\left(\begin{array}{c}
v_{1}^{2}-v_{2}^{2} \\
2 v_{1} v_{2}
\end{array}\right) \\
& =\left(v_{1}, v_{2}\right)\left(\begin{array}{cc}
a x_{1}+b x_{2} & -b x_{1}+a x_{2} \\
-b x_{1}+a x_{2} & -a x_{1}-b x_{2}
\end{array}\right)\left(\begin{array}{l}
v_{1} \\
v_{2}
\end{array}\right) .
\end{aligned}
$$

A simple inspection will show that the last $2 \times 2$ matrix is precisely what it is claimed to be in formula (5.13). The eigenvalues are readily computed.

REMARK 5.5. The function $f(z):=1 /(1+z)$ is a holomorphic bijection of $\mathbb{C} \backslash\{-1\}$ onto $\mathbb{C} \backslash\{0\}$. We have that $z f^{\prime \prime}(z) / f^{\prime}(z)=-2 z /(1+z)$ and

$$
\begin{aligned}
\left|f^{\prime}(z)\right| \geqslant\left|z f^{\prime \prime}(z)\right| & \Longleftrightarrow\left|z-\frac{1}{3}\right| \leqslant \frac{2}{3} \Longleftrightarrow f(z) \in\{w \in \mathbb{C}:|w-1| \leqslant 1 / 2\}, \\
\Re\left(1+\frac{z f^{\prime \prime}(z)}{f^{\prime}(z)}\right)>0 \Longleftrightarrow|z|<1 & \Longleftrightarrow f(z) \in\{w \in \mathbb{C}: \Re w>1 / 2\} .
\end{aligned}
$$

The images of the disks centred at the origin are

$$
\begin{aligned}
& f\left(D_{r}\right)=\left\{w \in \mathbb{C}:\left|w-\frac{1}{1-r^{2}}\right|<\frac{r}{1-r^{2}}\right\} \quad \text { if } 0<r<1, \\
& f\left(D_{r}\right)=\{w \in \mathbb{C}: \Re w>1 / 2\} .
\end{aligned}
$$

The largest radius $r$ for which $w \mapsto\left|f^{-1}(w)\right|^{2}$ is convex on $f\left(D_{r}\right)$ is $r=1 / 3$. However that real function is convex on the image (which happens to be convex) of the larger 
disk centred in $1 / 3$ with radius $2 / 3$, and it is not even locally convex elsewhere. In particular, for $1 / 3<r \leqslant 1$ the image $f\left(D_{r}\right)$ is a convex set although the function $w \mapsto\left|f^{-1}(w)\right|^{2}$ is not convex on it.

Before starting the proof of Proposition 1.5, we recall a few elementary facts from the theory of real functions of one variable. Let $I$ be a nonempty interval of $\mathbb{R}$.

(1) A function with derivative at every point of $I$ is convex on $I$ if and only if its derivative is weakly increasing on $I$.

(2) A function $\psi: I \rightarrow \mathbb{R}$ is said to be (weakly) increasing at $t_{0} \in I$ if $\left(\psi(t)-\psi\left(t_{0}\right)\right)\left(t-t_{0}\right) \geqslant 0$ for all $t \in I$ close enough to $t_{0}$.

(3) A real function is weakly increasing on $I$ if and only if it is weakly increasing at every point of $I$.

ProOF of Proposition 1.5: The simple injectivity can be proved for larger radii with the technique of the local inverse function theorem. Define in fact $\tilde{f}(x):=$ $f^{\prime}\left(x_{0}\right)^{-1} f(x)$. If $x \in B_{r}\left(x_{0}\right)$ we have

$$
\left|\widetilde{f}^{\prime}(x)-I\right|=\left|\left(f^{\prime}\left(x_{0}\right)^{-1}-f^{\prime}(x)^{-1}\right) f^{\prime}(x)\right| \leqslant L\left|x-x_{0}\right| \frac{1}{M_{1}} \leqslant \frac{L r}{M_{1}} .
$$

The function $x \mapsto \tilde{f}(x)-x$ is then Lipschitz continuous on $B_{r}\left(x_{0}\right)$ with constant $L r / M_{1}$ and if $x^{\prime}, x^{\prime \prime} \in B_{r}\left(x_{0}\right)$ we can write

$$
\begin{aligned}
\left|\tilde{f}\left(x^{\prime}\right)-\tilde{f}\left(x^{\prime \prime}\right)\right| & \geqslant\left|x^{\prime}-x^{\prime \prime}\right|-\left|\left(\tilde{f}\left(x^{\prime}\right)-x^{\prime}\right)-\left(\tilde{f}\left(x^{\prime \prime}\right)-x^{\prime \prime}\right)\right| \\
& \geqslant\left|x^{\prime}-x^{\prime \prime}\right|-\frac{L r}{M_{1}}\left|x^{\prime}-x^{\prime \prime}\right|=\left(1-\frac{L r}{M_{1}}\right)\left|x^{\prime}-x^{\prime \prime}\right| .
\end{aligned}
$$

Hence $\tilde{f}$, and $f$ too, are one-to one on $B_{r}\left(x_{0}\right)$ whenever $0<r<M_{1} / L$. It is obvious that the injectivity extends to the limit case $r=M_{1} / L$. Notice that $M_{1}^{2} / L M_{2} \leqslant$ $M_{1} / M_{2}$ because $M_{1} \leqslant M_{2}$. Let us proceed to prove that when $r \leqslant M_{1}^{2} / L M_{2}$ the function $y \mapsto\left|f^{-1}(y)-x_{0}\right|^{2}$ is locally convex. Let $\widetilde{k}(x):=\left|x-x_{0}\right|^{2}$. It is enough to show that the first derivative of the $C^{1}$ function $t \mapsto\left(\tilde{k} \circ f^{-1}\right)(x+t u)$, defined in a neighbourhood of $0 \in \mathbb{R}$, is pointwise increasing at $t=0$ for any choice of $x \in B_{r}\left(x_{0}\right)$, $u \in \mathbb{R}^{n}$. Let $\gamma(t):=f^{-1}(x+t u)$. We have

$$
\begin{aligned}
h^{\prime}(t) & =\frac{d}{d t} \widetilde{k}(\gamma(t))=\widetilde{k}^{\prime}(\gamma(t))\left(\gamma^{\prime}(t)-\gamma^{\prime}(0)\right)+\widetilde{k}^{\prime}(\gamma(t)) \gamma^{\prime}(0) \\
& =2\left(\gamma(t)-x_{0}\right) \cdot\left(\gamma^{\prime}(t)-\gamma^{\prime}(0)\right)+2\left(\gamma(t)-x_{0}\right) \cdot \gamma^{\prime}(0) .
\end{aligned}
$$

The term $\left(\gamma(t)-x_{0}\right) \cdot \gamma^{\prime}(0)$ is differentiable and for $t=0$ we can write

$$
\left.\frac{d}{d t}\left(\gamma(t)-x_{0}\right) \cdot \gamma^{\prime}(0)\right|_{t=0}=\left|\gamma^{\prime}(0)\right|^{2}=\left|f^{\prime}(x)^{-1} u\right|^{2} \geqslant M_{1}^{2}|u|^{2} .
$$


In particular, for any $\varepsilon>0$ and $|t|$ small enough we have

$$
2\left(\gamma(t)-x_{0}\right) \cdot \gamma^{\prime}(0) \begin{cases}\geqslant 2(1-\varepsilon) M_{1}^{2}|u|^{2} t & \text { for small } t \geqslant 0 \\ \leqslant 2(1-\varepsilon) M_{1}^{2}|u|^{2} t & \text { for small } t<0 .\end{cases}
$$

The rest of the assumptions (1.5) can be used this way:

$$
\begin{array}{r}
\left|\gamma^{\prime}(t)-\gamma^{\prime}(0)\right| \leqslant\left|\left(f^{\prime}(\gamma(t))^{-1}-f^{\prime}(x)^{-1}\right) u\right| \leqslant L|\gamma(t)-x||u| \\
=L|u|\left|\int_{0}^{t} \gamma^{\prime}(s) d s\right| \leqslant L|u|\left|\int_{0}^{t}\right| \gamma^{\prime}(s)|d s| \\
=L|u|\left|\int_{0}^{t}\right| f^{\prime}(\gamma(s))^{-1} u|d s| \leqslant L M_{2}|u|^{2}|t| .
\end{array}
$$

If we choose $\varepsilon<1-\left|x-x_{0}\right| / r<1$ we have that $\left|\gamma(t)-x_{0}\right| \leqslant(1-\varepsilon) r$ for any $|t|$ small enough. For those $t$ we can estimate the remaining term in (5.20) as follows:

$$
\left|2\left(\gamma(t)-x_{0}\right) \cdot\left(\gamma^{\prime}(t)-\gamma^{\prime}(0)\right)\right| \leqslant 2(1-\varepsilon) r L M_{2}|u|^{2}|t| .
$$

Relations (5.21) and (5.24) together give, for such choices of $\varepsilon$,

$$
h^{\prime}(t) \begin{cases}\geqslant 2(1-\varepsilon)\left(M_{1}^{2}-r L M_{2}\right)|u|^{2} t & \text { for small } t \geqslant 0, \\ \leqslant 2(1-\varepsilon)\left(M_{1}^{2}-r L M_{2}\right)|u|^{2} t & \text { for small } t<0 .\end{cases}
$$

This shows that $h^{\prime}(t)$ is pointwise increasing at $t=0$ whenever $r \leqslant M_{1}^{2} / L M_{2}$.

Finally, to prove that $f\left(B_{r}\left(x_{0}\right)\right)$ is convex, apply Theorem 1.1 with the auxiliary function $k(x):=1 /\left(r^{2}-\widetilde{k}(x)\right)$, as in the proof of Proposition 1.3.

REMARK 5.6. If we are looking for a homeomorphism $f$ defined in a neighbourhood of the origin $x_{0} \in \mathbb{R}^{n}$ for which the function $y \mapsto\left|f^{-1}(y)-x_{0}\right|^{2}$ is not convex near $f\left(x_{0}\right)$, it will be enough if $f$ maps every ball $B_{r}\left(x_{0}\right)$ with small radius $r$ onto a nonconvex set. For example, in two dimensions we can impose that $f$ maps $B_{r}(0)$ onto the hour-glass shaped sets $0 \leqslant \rho<\left(1+\sin ^{2} \theta\right) r$ (in polar coordinates) by defining

$$
f\left(\begin{array}{l}
x \\
y
\end{array}\right)= \begin{cases}\frac{x^{2}+2 y^{2}}{x^{2}+y^{2}}\left(\begin{array}{l}
x \\
y
\end{array}\right) & \text { if }(x, y) \neq(0,0) \\
\left(\begin{array}{l}
0 \\
0
\end{array}\right) & \text { if }(x, y)=(0,0) .\end{cases}
$$

This $f: \mathbb{R}^{2} \rightarrow \mathbb{R}^{2}$ is obviously continuous outside the origin and, being homogeneous of degree 1 , it is continuous also at the origin. The inverse $f^{-1}$ exists over all of $\mathbb{R}^{2}$ 
and it is continuous everywhere too. Hence $f$ is a homeomorphism. The first partial derivatives of $f$ and $f^{-1}$ exist outside the origin and they are nonconstant, continuous and homogenous of degree 0 . Hence they are bounded but they have no limit at the origin. Then both $f$ and $f^{-1}$ are globally Lipschitz continuous but neither is $C^{1}$.

To build a possible $C^{1}$ example we cannot insist that the images of the balls centred in the origin be homothetic to each other. Let us try by making the size of the dent in the set $f\left(B_{r}(0,0)\right)$ to be of order $o(r)$, but with an increasingly pointed shape, as $r$ decreases to 0 . Let $\varphi: \mathbb{R} \rightarrow \mathbb{R}$ be a $C^{\infty}$ function such that

$$
\begin{gathered}
\varphi(-t)=\varphi(t) \quad \text { for all } t \in \mathbb{R} \\
\varphi(t) \equiv 1 \quad \text { for }|t| \geqslant 1, \varphi(0)=0, \quad \varphi^{\prime}(t)>0 \quad \text { for } 0<t<1, \\
\varphi^{\prime \prime}(0)>1 .
\end{gathered}
$$

Let

$$
h(\rho, \theta):= \begin{cases}\rho+\rho^{\alpha} \varphi\left(\theta \rho^{-\beta}\right) & \text { if } \rho>0 \\ 0 & \text { if } \rho=0\end{cases}
$$

where $\alpha, \beta>0$ are parameters to be adjusted. We define our $f: \mathbb{R}^{2} \rightarrow \mathbb{R}^{2}$ in polar coordinates as

$$
(\rho, \theta) \mapsto(h(\rho, \theta), \theta) \quad \text { for } \rho \geqslant 0, \theta \in(-\pi, \pi] .
$$

This $f$ is designed to transform the circle $\rho=r=$ constant into a curve which, for small radii $r$, is essentially the circle centred at the origin and radius $r+r^{\alpha}$, except for a perturbation located around $\theta=0$ (more precisely, restricted to $|\theta| \leqslant r^{\beta}$ ), where the distance from the origin dips to reach the minimum value $r$ at $\theta=0$. We wish the dent to be deep enough to make that curve the boundary of a nonconvex bounded set; on the other hand, if we overdo it we risk disrupting the differentiability of $f$.

We shall omit the calculations that show that we can indeed strike a balance: it happens when $\alpha>0, \beta>0, \alpha-2 \beta \leqslant 1$ and $\alpha-\beta>1$. A possible particular choice of parameters is $\alpha=3 / 2, \beta=1 / 4$.

REMARK 5.7. Some recent papers [14], [6], [15] by the authors proved sufficient conditions for a local diffeomorphism to be one-to-one, conditions that were expressed in terms of an "auxiliary function" $k$. For our present purposes those results can be stated as follows:

Let $\Omega$ be a nonempty, open and connected subset of $\mathbb{R}^{n}, x_{0} \in \Omega, f: \Omega \rightarrow \mathbb{R}^{n}$ be a $C^{1}$ local diffeomorphism and $k: \Omega \rightarrow \mathbb{R}$ be a proper and bounded from below $C^{1}$ function. Then:

(a) if the quantity $k^{\prime}(x) f^{\prime}(x)^{-1}\left(f(x)-f\left(x_{0}\right)\right)$ is bounded from below on $\Omega$, then $f$ is one-to-one; 
(b) if $f\left(x_{0}\right)<c<+\infty$ and

$$
k^{\prime}(x) f^{\prime}(x)^{-1}\left(f(x)-f\left(x_{0}\right)\right)>0 \quad \text { for all } x \in \Omega \text { such that } k(x)=c,
$$

then $f$ is one-to-one on the connected component $C$ of $\{x \in \Omega$ : $k(x) \leqslant c\}$ containing $x_{0}$.

Suppose that the triple $(\Omega, f, k)$ satisfies Theorem 1.1, and that moreover $f$ is a $C^{1}$ local (hence global) diffeomorphism onto the convex set $f(\Omega)$, and that $k$ is $C^{\mathbf{1}}$. Pick any point $x_{0} \in \Omega$. Let us see whether the results (a) or (b) just quoted apply to the quadruple $\left(\Omega, f, k, x_{0}\right)$. This is not to be construed as an alternative proof of Theorem 1.1, because we assume it known from the start.

The function $K:=k \circ f^{-1}$ is $C^{1}$ and convex on the convex set $f(\Omega)$. If we subtract from it its differential function at the point $f\left(x_{0}\right)$ we are left with another convex function

$$
h(y):=K(y)-K^{\prime}\left(f\left(x_{0}\right)\right)\left(y-f\left(x_{0}\right)\right)
$$

which attains a minimum at $y=f\left(x_{0}\right)$ because the differential vanishes at that point. If we evaluate $h$ along the straight line $\tau \mapsto(1-\tau) f\left(x_{0}\right)+\tau f(x)$ that joins $f\left(x_{0}\right)$ with a generic point $f(x)$ in the convex set $f(\Omega)$, we get a convex function of $\tau$, defined in an open interval containing $[0,1]$, with a minimum at $\tau=0$. Hence the derivative at $\tau=1$ will be $\geqslant 0$ :

$$
\begin{aligned}
0 & \leqslant \frac{d}{d \tau} h\left((1-\tau) f\left(x_{0}\right)+\tau f(x)\right) \\
& =K^{\prime}(f(x))\left(f(x)-f\left(x_{0}\right)\right)-K^{\prime}\left(f\left(x_{0}\right)\right)\left(f(x)-f\left(x_{0}\right)\right) .
\end{aligned}
$$

Since $K^{\prime}(f(x))=k^{\prime}(x) f^{\prime}(x)^{-1}$, we deduce that

$$
\begin{aligned}
k^{\prime}(x) f^{\prime}(x)^{-1}\left(f(x)-f\left(x_{0}\right)\right) & =K^{\prime}(f(x))\left(f(x)-f\left(x_{0}\right)\right) \\
& \geqslant K^{\prime}\left(f\left(x_{0}\right)\right)\left(f(x)-f\left(x_{0}\right)\right) \\
& \geqslant-\left\|K^{\prime}\left(f\left(x_{0}\right)\right)\right\|\left|f(x)-f\left(x_{0}\right)\right|
\end{aligned}
$$

for all $x \in \Omega$. Thus condition (a) of the quoted statements holds on the supplementary assumption that $f(\Omega)$ be bounded.

Next, choose any constant $c>f\left(x_{0}\right)$ and let $x \in \Omega$ be such that $k(x)=c$ (such an $x$ surely exists because $k(\Omega)$ is an interval and $\sup k(\Omega)=+\infty$ ). The set $\{z \in \Omega: k(z) \leqslant c\}$ is connected because it is the image through $f^{-1}$ of the convex set $\left\{y \in f(\Omega): k \circ f^{-1}(y) \leqslant c\right\}$. Along the straight line that joins $f\left(x_{0}\right)$ with $f(x)$ the 
function $K$ is $C^{1}$, convex, and at $f(x)$ it attains a higher value than at $f\left(x_{0}\right)$. Hence the derivative of

$$
\tau \mapsto K\left((1-\tau) f\left(x_{0}\right)+\tau f(x)\right)
$$

will be positive at $\tau=1$, corresponding to $f(x)$. Again by means of the formula for $K^{\prime}(f(x))$ we get

$$
\begin{gathered}
0<\frac{d}{d \tau}\left(\left.K\left((1-\tau) f\left(x_{0}\right)+\tau f(x)\right)\right|_{\tau=1}=K^{\prime}(f(x))\left(f(x)-f\left(x_{0}\right)\right)\right. \\
\quad=k^{\prime}(x) f^{\prime}(x)^{-1}\left(f(x)-f\left(x_{0}\right)\right)
\end{gathered}
$$

for all $x \in \Omega$ such that $k(x)=c$, which is equivalent to condition (b).

\section{Motivation from Differential Geometry}

Let $\Omega$ be a nonempty, open and connected subset of $\mathbb{R}^{n}$, regarded as a $C^{\infty}$ manifold with the atlas given by the canonical co-ordinates of $\mathbb{R}^{n}$ and the trivial tangent bundle $\Omega \times \mathbb{R}^{n}$. Let $f: \Omega \rightarrow \mathbb{R}^{n}$ be a $C^{\infty}$ local diffeomorphism, and let us also regard the image $f(\Omega)$ as a $C^{\infty}$ manifold in the same way, but here we endow it with the Riemannian structure induced by the canonical scalar product of $\mathbb{R}^{n}$.

There is one and only one Riemannian structure on $\Omega$ for which the length of a smooth curve $\gamma: I \rightarrow \Omega$ in $\Omega$ coincides with the length of the transformed curve $f \circ \gamma$ in $f(\Omega)$ : the inner product $\left\langle u_{x}, v_{x}\right\rangle_{x}$ of two vectors $u_{x}, v_{x}$ in the tangent fibre at $x \in \Omega$ must be

$$
\left\langle u_{x}, v_{x}\right\rangle_{x}:=\left(f^{\prime}(x) u_{x}\right) \cdot\left(f^{\prime}(x) v_{x}\right),
$$

where on the right-hand side we have the canonical scalar product of $\mathbb{R}^{n}$. This is indeed a Riemannian metric because $f^{\prime}(x)$ is an isomorphism. After all, $f$ can also be regarded as a change of co-ordinates in a neighbourhood of any point. In canonical co-ordinates the metric tensor will be represented by the matrix $f^{\prime}(x)^{T} f^{\prime}(x)$, where $f^{\prime}(x)$ is seen as an $n \times n$ matrix and ${ }^{T}$ denotes transposition. This tensor is called the Cauchy-Green strain tensor in continuum mechanics.

When $\Omega$ and $f(\Omega)$ are given these structures, the map $f$ will transform geodesic curves in $\Omega$ into geodesic curves in $f(\Omega)$. The latter are trivially straight lines with affine parameterisation: $t \mapsto f(x)+t v$. The geodesic curves in $\Omega$ are simply the linelifting curves of Section 2 (plus smoothness of $f$ ), or, equivalently, the solutions of Ważewski's equation (2.2).

Suppose that any two points $x_{0}, x_{1} \in \Omega$ can be connected by a geodesic line $\gamma$ in $\Omega$. Then $f(\Omega)$ is convex, because the images $f\left(x_{0}\right)$ and $f\left(x_{1}\right)$ are connected by the 
straight line $f \circ \gamma$ in $f(\Omega)$. Moreover, if $f\left(x_{0}\right)=f\left(x_{1}\right)$, then $f$ is constant along $\gamma$, which can only happen when $\gamma$ itself is constant, because $f$ is locally invertible. Hence $f$ must also be globally one-to-one.

Now, whatever hypothesis on a Riemannian manifold that ensures that any pair of points are connected by a geodesic arc can be translated into a condition on (the metric induced by) the map $f$ that will imply global invertibility and convex image. The starting point of the research that produced this paper was the following theorem of Gordon's (see [5]):

THEOREM. Let $M$ be a connected Riemannian manifold. If there exists a $C^{\infty}$ function $k: M \rightarrow \mathbb{R}$ which is proper, bounded from below and geodesically convex (that is, the second covariant differential of $k$ is positive semidefinite, or, equivalently, $(k \circ \gamma) " \geqslant 0$ for every geodesic arc $\gamma$ in $M)$, then any two points of $M$ can be connected by a geodesic arc.

Within the Riemannian structure introduced on $\Omega$, it is straightforward to verify that the condition of geodesic convexity on $k$ is simply our Proposition 1.2, and the second covariant differential of $k$ is precisely the quadratic form $Q_{x}$ of formula (1.1).

\section{REFERENCES}

[1] S. Banach and S. Mazur, 'Über mehrdeutige stetige Abbildungen', Studia Math. 5 (1934), 174-178.

[2] F. Browder, 'Covering spaces, fiber spaces and local homeomorphisms', Duke Math. J. 21 (1954), 329-336.

[3] R. Caccioppoli, 'Sugli elementi uniti delle trasformazioni funzionali', Rend. Sem. Mat. Univ. Padova 3 (1932), 1-15.

[4] P.L. Duren, Univalent Functions (Springer-Verlag, Berlin, Heidelberg, New York, 1983).

[5] W.B. Gordon, 'The existence of geodesics joining two given points', J. Differential Geom. 9 (1974), 443-450.

[6] G. Gorni, 'A criterion of invertibility in the large for local diffeomorphisms between Banach spaces', Nonlinear Anal. 21 (1993), 43-47.

[7] J. Hadamard, 'Sur les transformations ponctuelles', Bull. Soc. Math. France 34 (1906), 71-84.

[8] M.P. Levy, 'Sur le fonctions de ligne implicites', Bull. Soc. Math. France 48 (1920), 13-27.

[9] G.H. Meisters and C. Olech, 'Global stability, injectivity, and the Jacobian conjecture', in Proc. of the First World Congress of Nonlinear Analysts, (Lakshmikantham, Editor) (Tampa, Florida, 1992) (to appear).

[10] T. Parthasarathy, On global univalence theorems, Lecture Notes in Math. 977 (Springer Verlag, Berlin, Heidelberg, New York, 1983).

[11] W.C. Rheinboldt, 'Local mapping relations and global implicit function theorems', Trans. Amer. Math. Soc. 138 (1969), 183-198. 
[12] J. Sotomayor, 'Inversion of smooth mappings', Z. Angew. Math. Phys. 41 (1990), 306-310.

[13] T. Ważewski, 'Sur l'evaluation du domain d'existence de fonctions implicites réelles ou complexes', Ann. Soc. Math. Polon. 20 (1947), 81-120.

[14] G. Zampieri, 'Finding domains of invertibility for smooth functions by means of attraction basins', J. Differential Equations 104 (1993), 11-19.

[15] G. Zampieri, 'Diffeomorphisms with Banach space domains', Nonlinear Anal. 19 (1992), 923-932.

[16] G. Zampieri and G. Gorni, 'On the Jacobian conjecture for global asymptotic stability', J. Dynamics Diff. Eq. 4 (1992), 43-55.

Dipartimento di Matematica Pura e Applicata Università di Padova

via Belzoni 7

35131 Padova

Italy

e-mail: Gaetano@pdmat1.unipd.it
Dipartimento di Matematica e Informatica Università di Udine

via Zanon 6

33100 Udine

Italy

e-mail: Gorni@udmi5400.cineca.it 